Fac. Vet. Med.,

Al-Baath Univ., Syria.

\title{
COMPARISON BETWEEN LAPAROSCOPIC VERSUS OPEN OVARIECTOMY IN DOGS
}

(With 3 Tables and 7 Figures)

مقارنة بين الجراحة التظيرية والجراحة المفتوحة في استئصال المبايض

\section{By}

برهان عساف ، محد موسى، أغر دعاس

(Received at 29/1/2011)

يهدف هذا البحث إلى وصف تقنية استئصلال المبايض عن طريق التنظير فى الكلاب

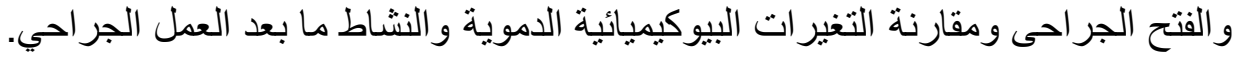
استخدم في هذا البحث 17 كلبة المجموعة الأولى (n=7) تم استئصال المبايض بطريقة الجراحة التنظيرية والمجموعة المئ

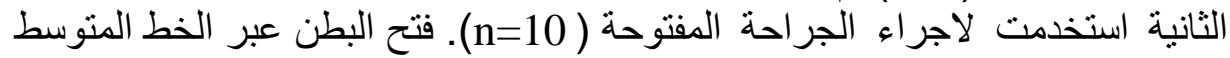

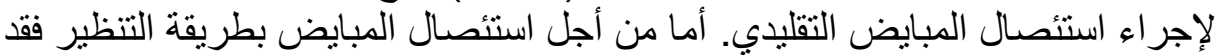

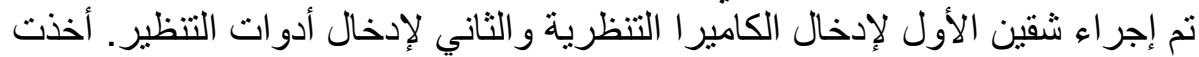

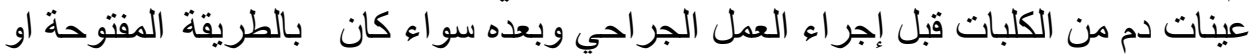

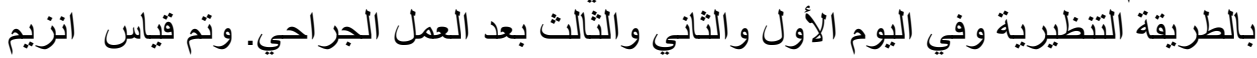

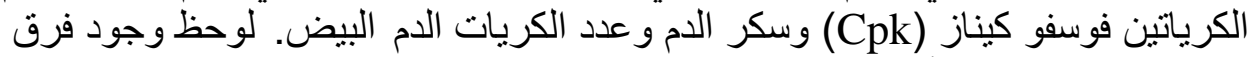

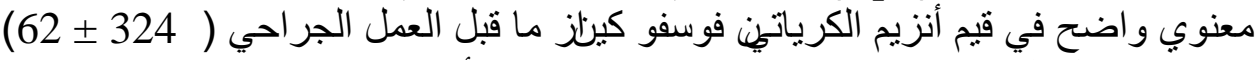

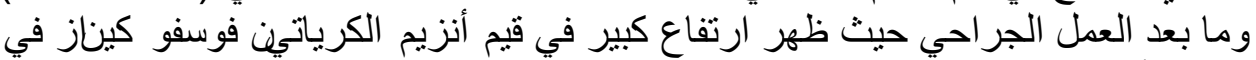

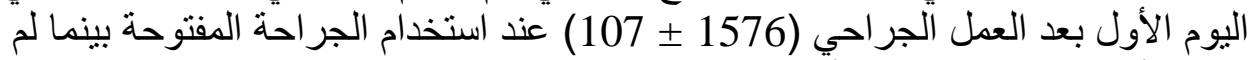

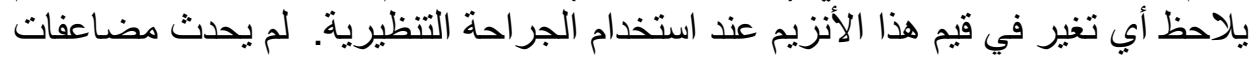

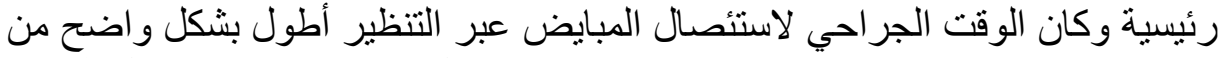

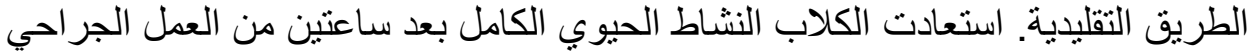
كلا

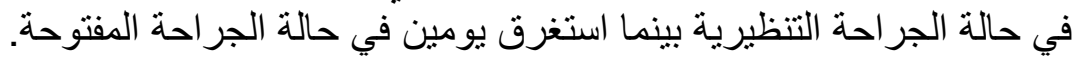

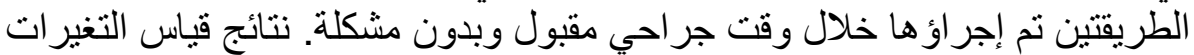
البيوكيميائية الدموية والنشاط الحيوي بعد العملية الجر احية كانت أفضل بطريقة الجراحة فئنة التنظيرية. 


\section{SUMMARY}

The present study aimed to describe a technique for laparoscopic ovariectomy in dogs, and compare the blood biochemical changes, the surgical time, complications, and postoperative activity of dogs undergoing laparoscopic ovariectomy to those undergoing traditional open ovariectomy. Intact breed female dogs $(n=17)$ were randomized. Ventral median celiotomy was performed for traditional open ovariectomy. A 2-midline portal technique using a laparoscope port and an instrument portal was used for laparoscopic ovariectomy. Blood samples were collected before and at the $1^{\text {th }}, 2^{\text {th }}, 3^{\text {th }}$ days after laparoscopic ovariectomy and traditional open ovariectomy. White blood cells count and biochemical analysis as blood Suger and Creatine Phosphokinase $(\mathrm{CpK})$ were performed. There is a significant different in the value of Creatine Phosphokinase $(\mathrm{CpK})$ between before $(324 \pm 62)$ and after $(1576 \pm 107)$ in the traditional open ovariectomy but no significant different in the laparoscopic ovariectomy. No major complications occurred and surgical time for laparoscopic ovariectomy was significantly longer than for traditional open ovariectomy $(\mathrm{P}<0.05)$. Dogs regeurate activity in the laparoscopic ovariectomy after two hours but in traditional open ovariectomy regeurate after two days. Both procedures were performed with reasonable surgical times and without major complication. Blood biochemical changes and postoperative activity was better in laparoscopic ovariectomy

Key words: Ovariectomy, dogs, laparoscopic ovariectomy, open ovariectomy.

\section{INTRODUCTION

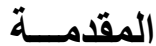

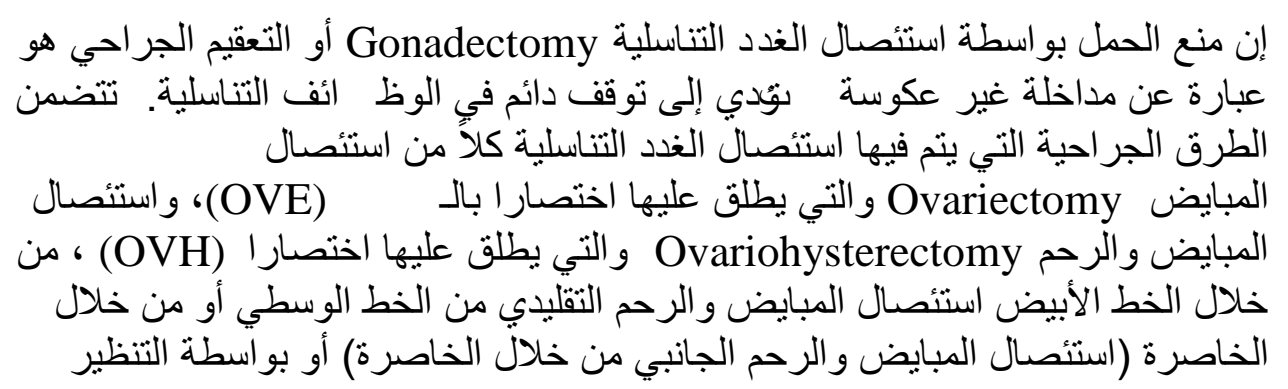


(Davidson et al., 2004; Devitt et al., 2005) التعقيم بو اسطة استئصال الأسهر Vasectomy أو استنصسال البوق Salpingectomy أو مبيض واحد تسبب وقف الوظيفة التناسلية ولكنها لا تلغي السلوك الكئه الجنسي أو حدوث الأمر اض الجنسية الناتجة عن الهرمونات الجنسية.

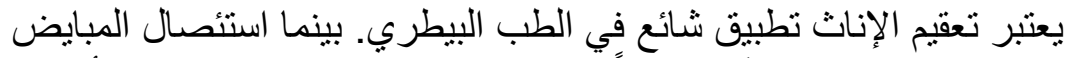

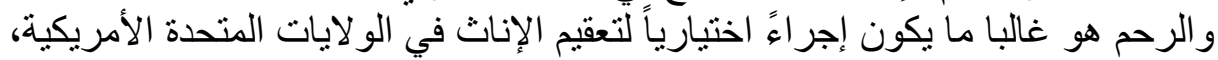

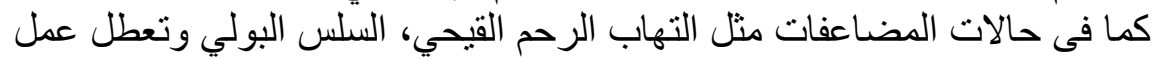

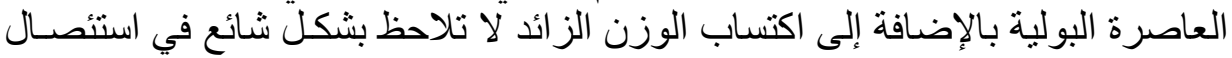

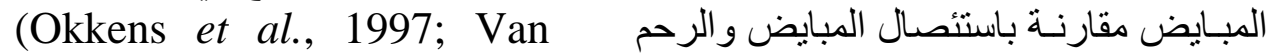
بالإضافة لذلك، فقد أنثار البعض إلى فوائد مرجّحة

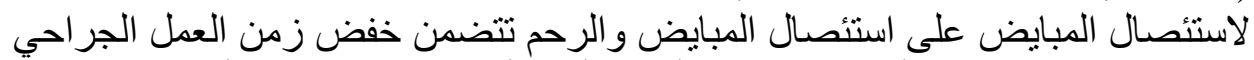

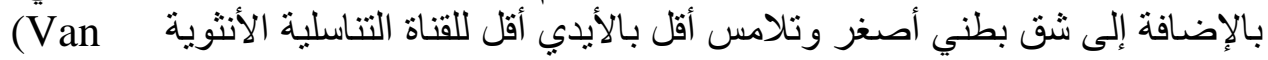
Goethem et al., 2003; Van Nimwegen et al., 2005; Goethem et al., .Van 2006) لا تزال تقنية التنظير جديدة في الطب البيطري و ولى كل حال فإنّ التعقيم

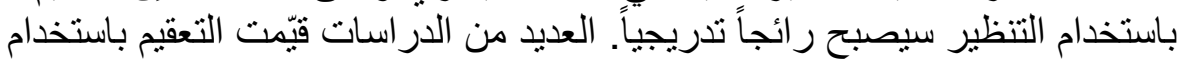

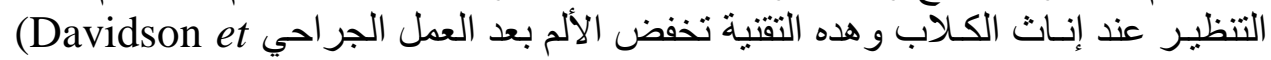
(Devitt et al., 2005) al., 2004; Hancock et al., 2005)

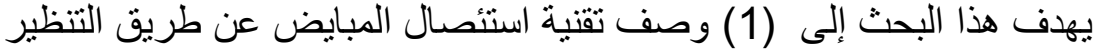

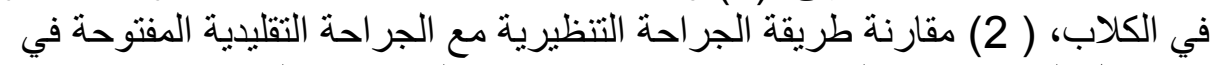

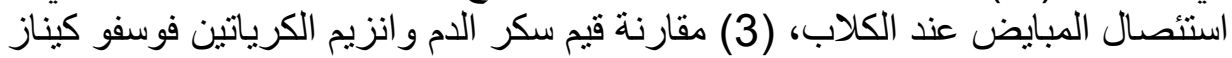

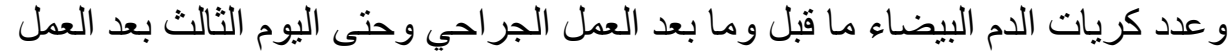

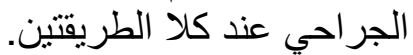

\section{MATERIALS and METHODS المـواد وطرق العمل}

$$
1
$$

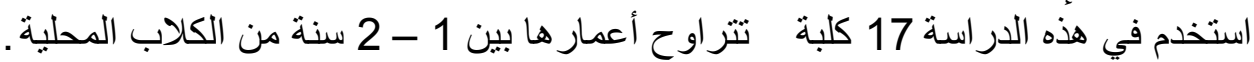

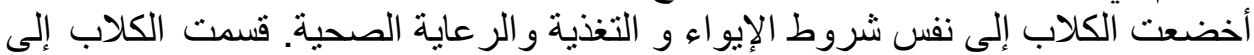

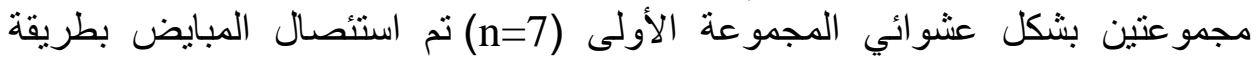

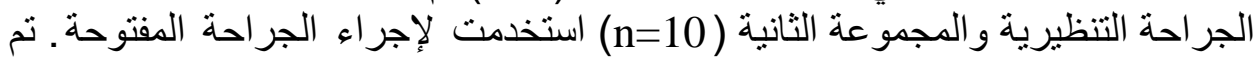
تصويم الحيو انات قبل 24 ساعة من بدء العمل الجر الجئ الجي. 
أجريت العمليات لجميع الكلاب في غرفة غرفة عمليات خاصة بالحيو انات الصغيرة تابعة

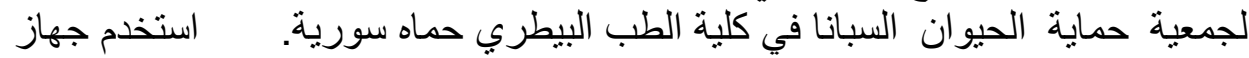
التخدير الاستنشاقي بالهالوثان لتخدير الحيو انات و بوجود طبيب تخدير خاص من قبل السبانا.

\section{3 - استئصال المبايض بالطريقة التقليدية عن طريق فتح البطن:}

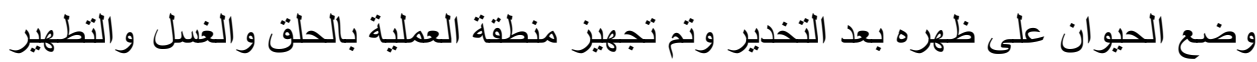

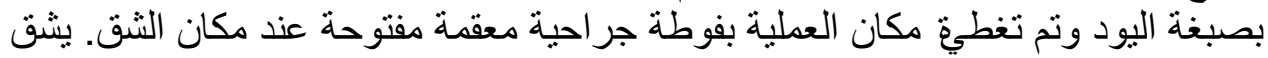

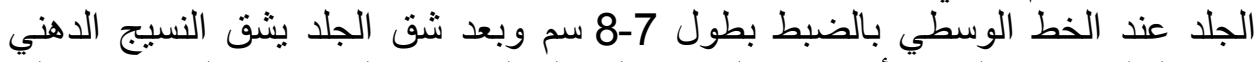

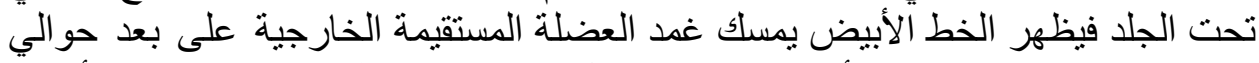

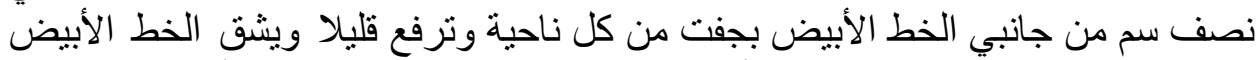

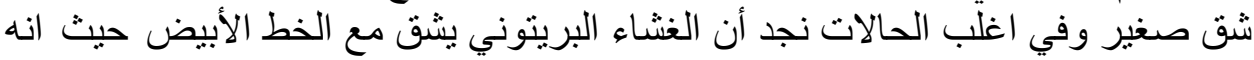

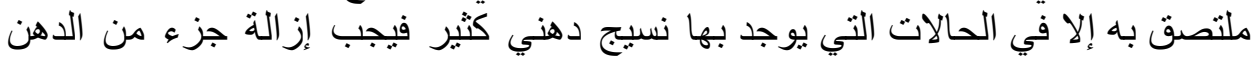

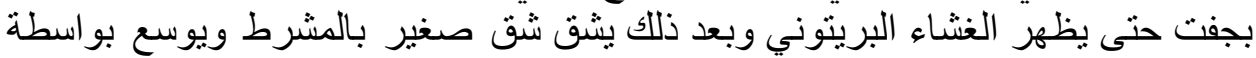

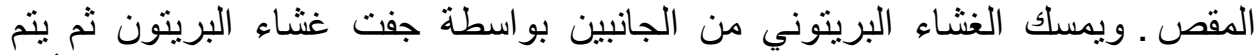

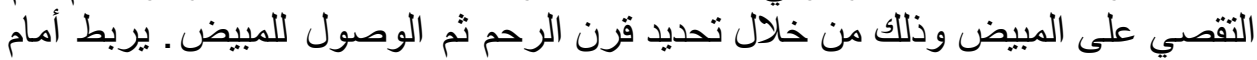

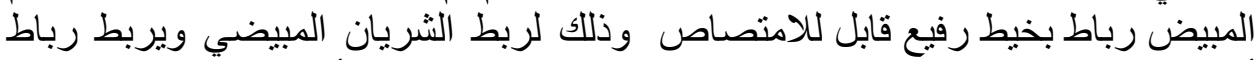

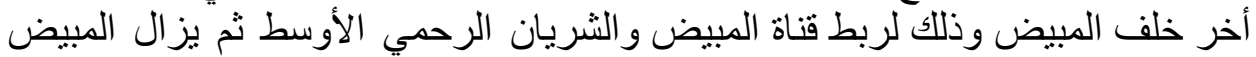

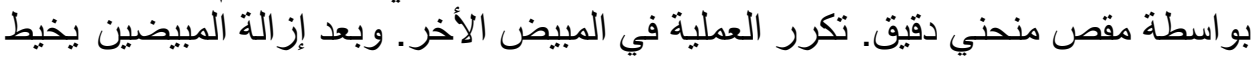

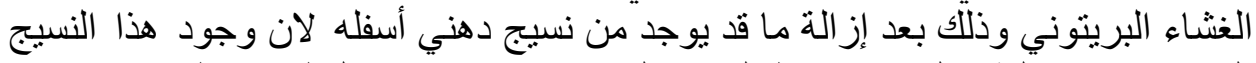

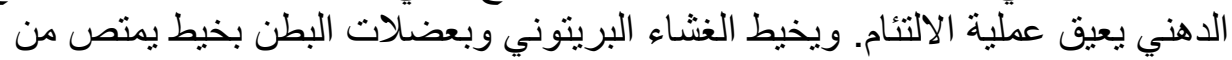

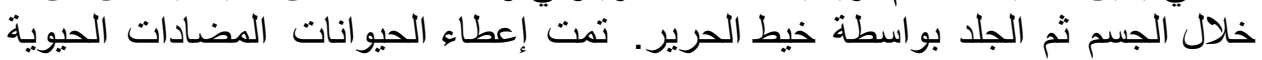
لمدة 5 أيام.

4 - استئصال المبايض بطريقة الجراحة التظظيرية:

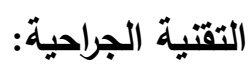

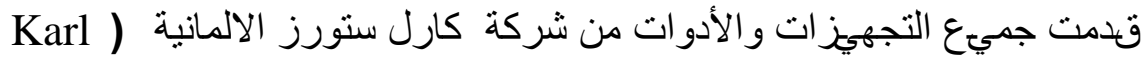
Storz Endoscopy, Goleta, CA البوابتين في استئصسال المب ايض بالجر احة التنظيرية ـ مُددت الكلبة بوضع الاستلقاء الظهري كما تم تثبيت بطنها وتهيئتها.

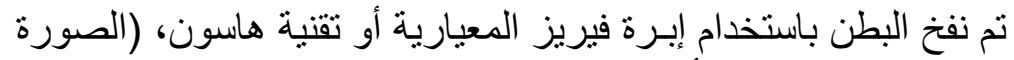

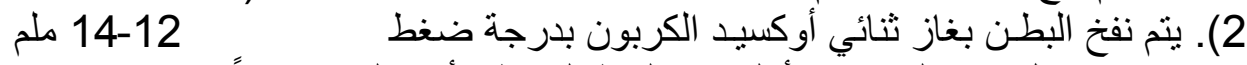

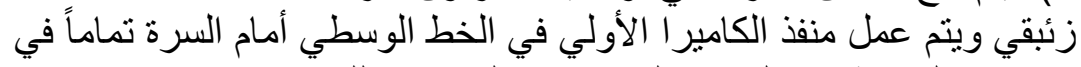

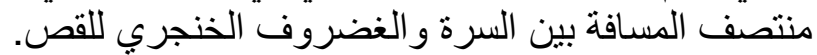

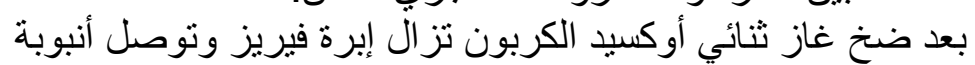

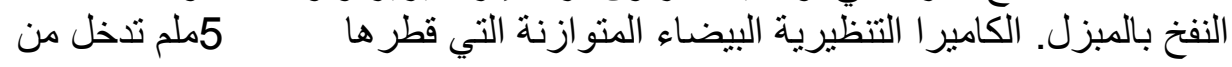


خلال المبزل (الصورة 3). تفحص المنطقة الموجودة أسفل المبزل تماماً لإيجاد أي ضرر.

ثم بنشأ الفتحة الذي تدخل منه الأدوات تحت النظر المباثر في منتصف

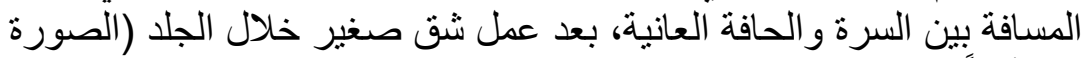

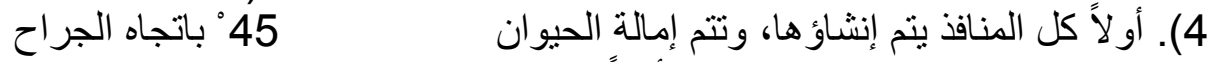

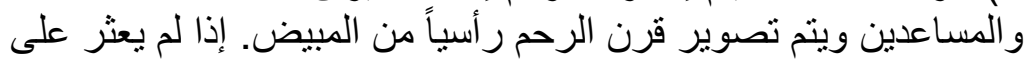

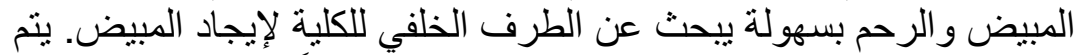

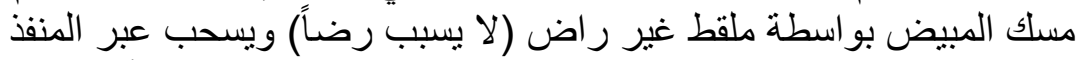

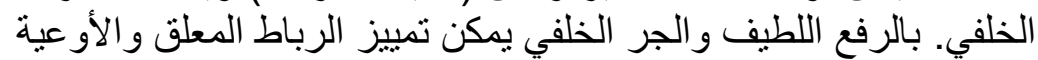

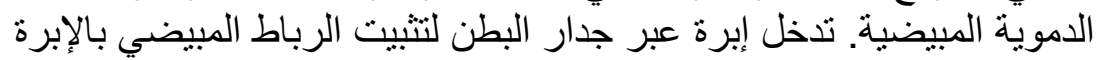
5

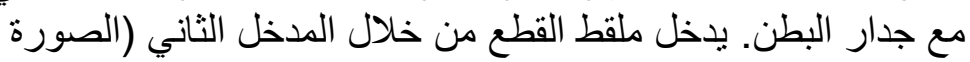
بعد ذللك كي الرباط المعلق و الثريان المبيضي في موقعين وبعد ذللك يتم قطعهما باستخدام مقص داخلي.

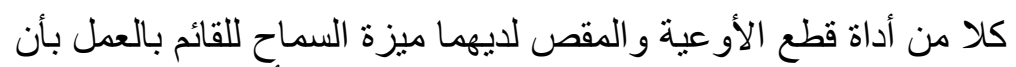

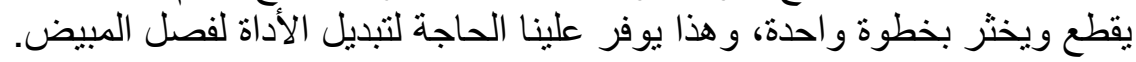

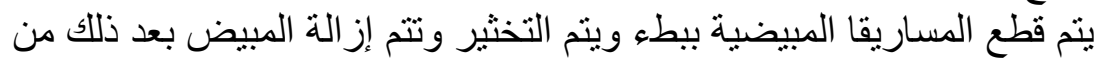

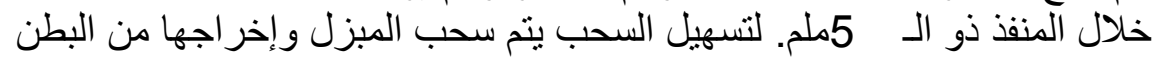

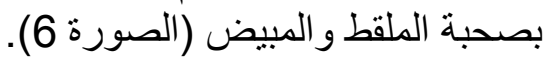

يدار الحيوان 45 بُ بالاتجاه المقابل ويز ال المبيض الأيمن بنفس الطريقة

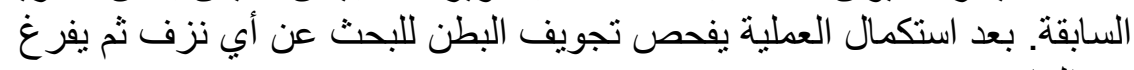
من الغاز.

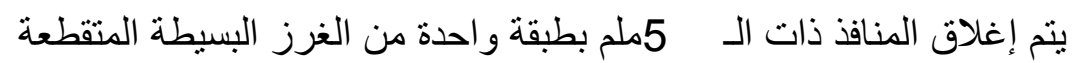

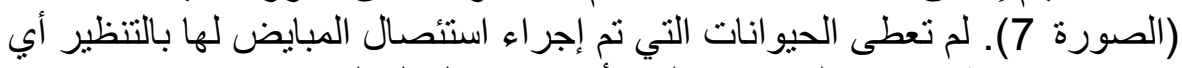

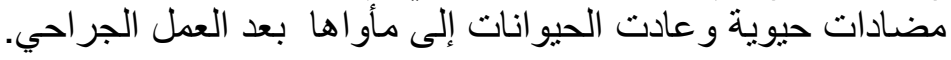


Assiut Vet. Med. J. Vol. 57 No. 129 April 2011

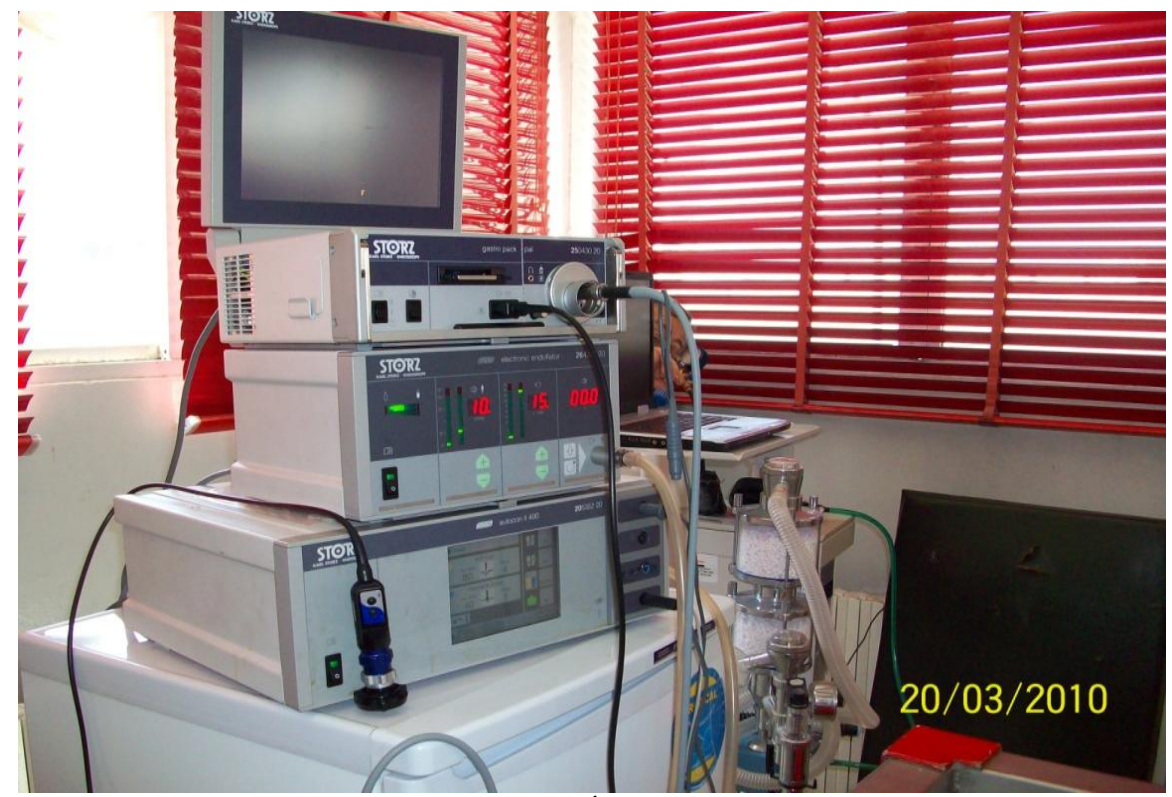

الصورة (1): صورة تظهر أجزاء جهاز التظير مع الوصلات.

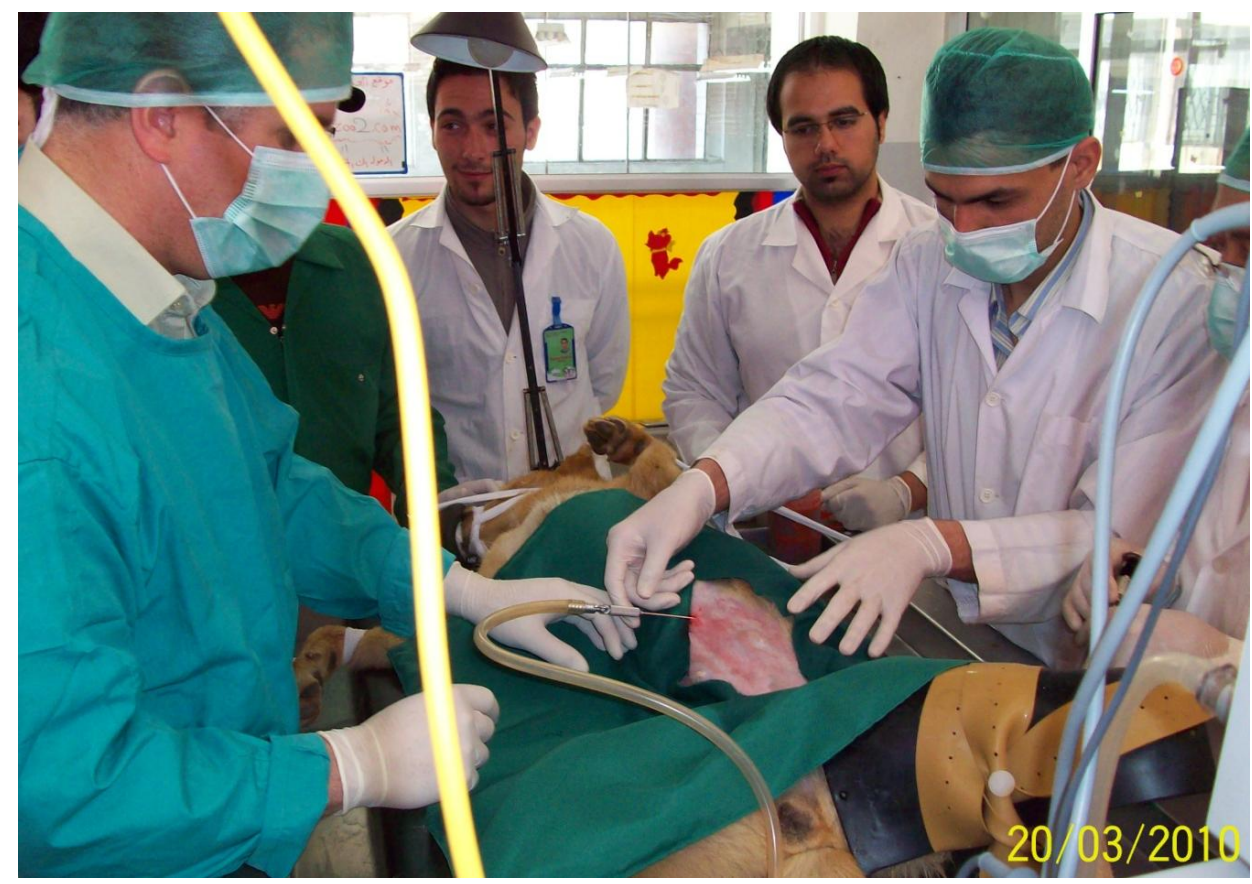

الصورة (2): إدخال الإبرة فيريز وضخ غاز ثاني أوكسيد الكربون 
Assiut Vet. Med. J. Vol. 57 No. 129 April 2011

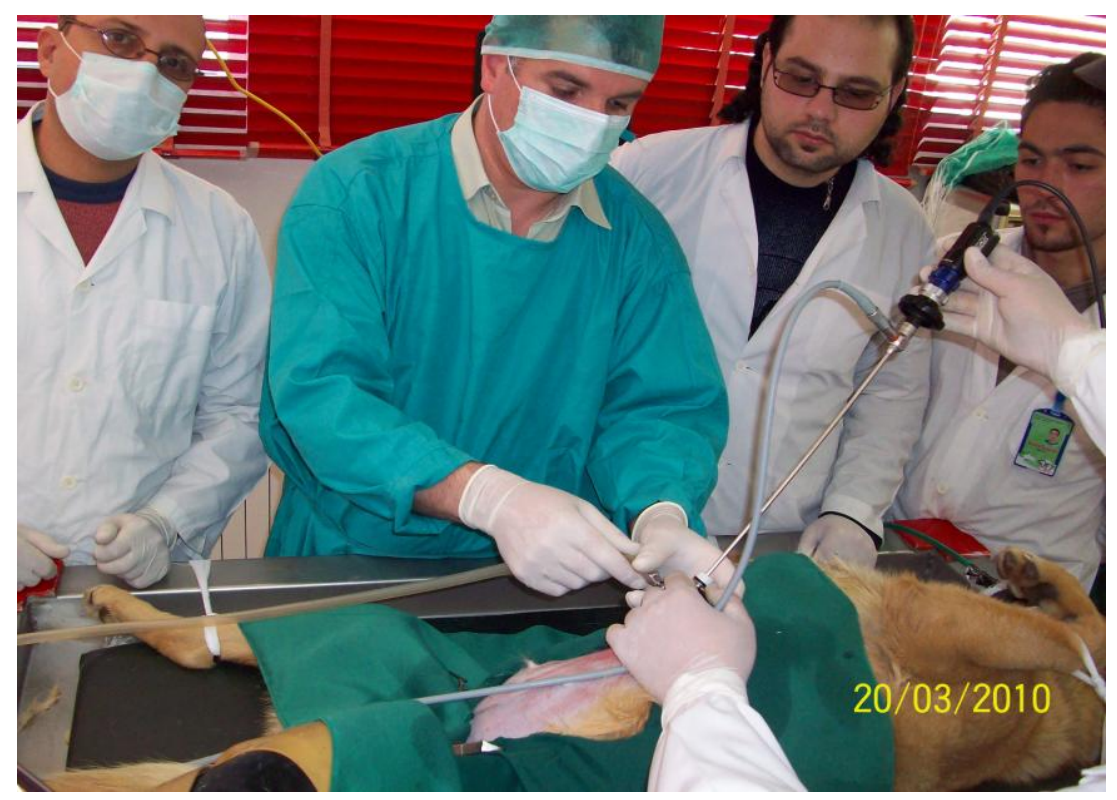

الصورة (3): ادخال المبزل بعد سحب ابرة فيريز في نفس الثقب وصله مع مضخة غاز ثاني أوكسيد الكربون.

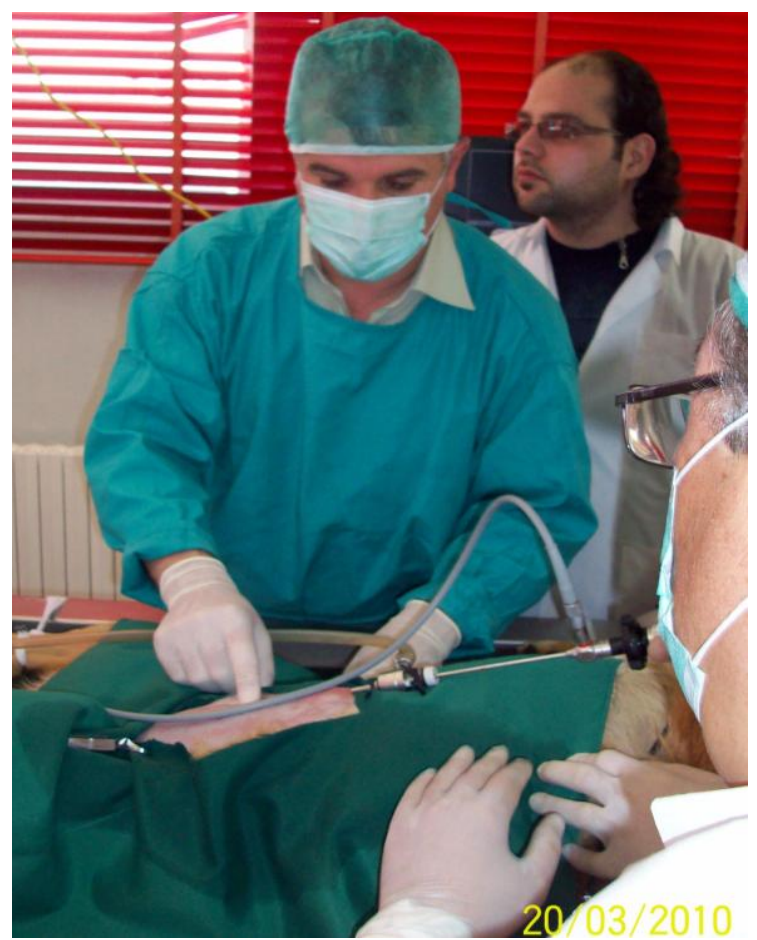

الصورة (4): إجراء شق صغير من أجل عمل فتحة ثانية لإدخال الأدوات تحت النظر

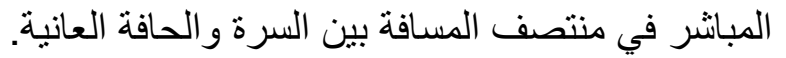


Assiut Vet. Med. J. Vol. 57 No. 129 April 2011
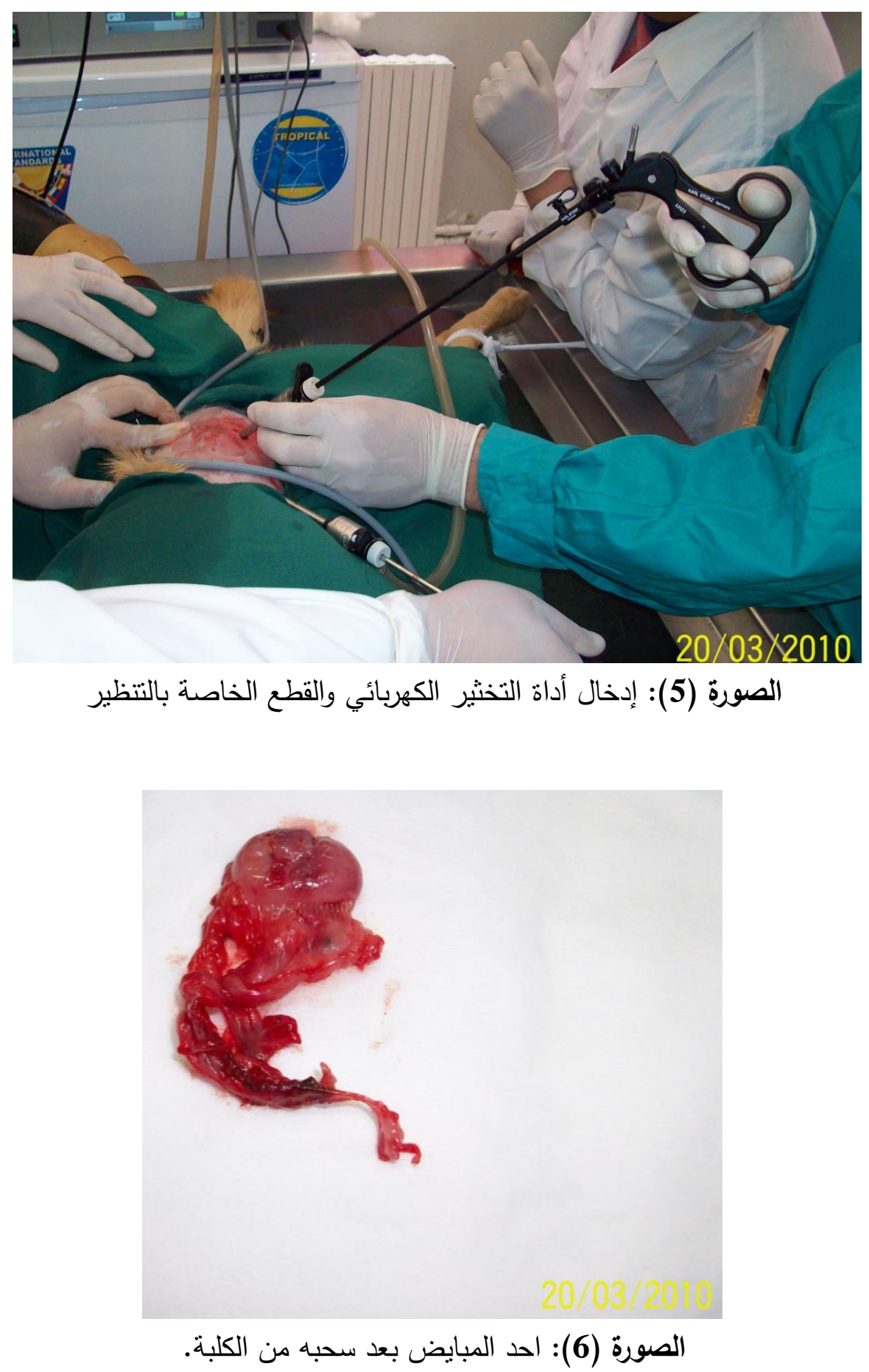


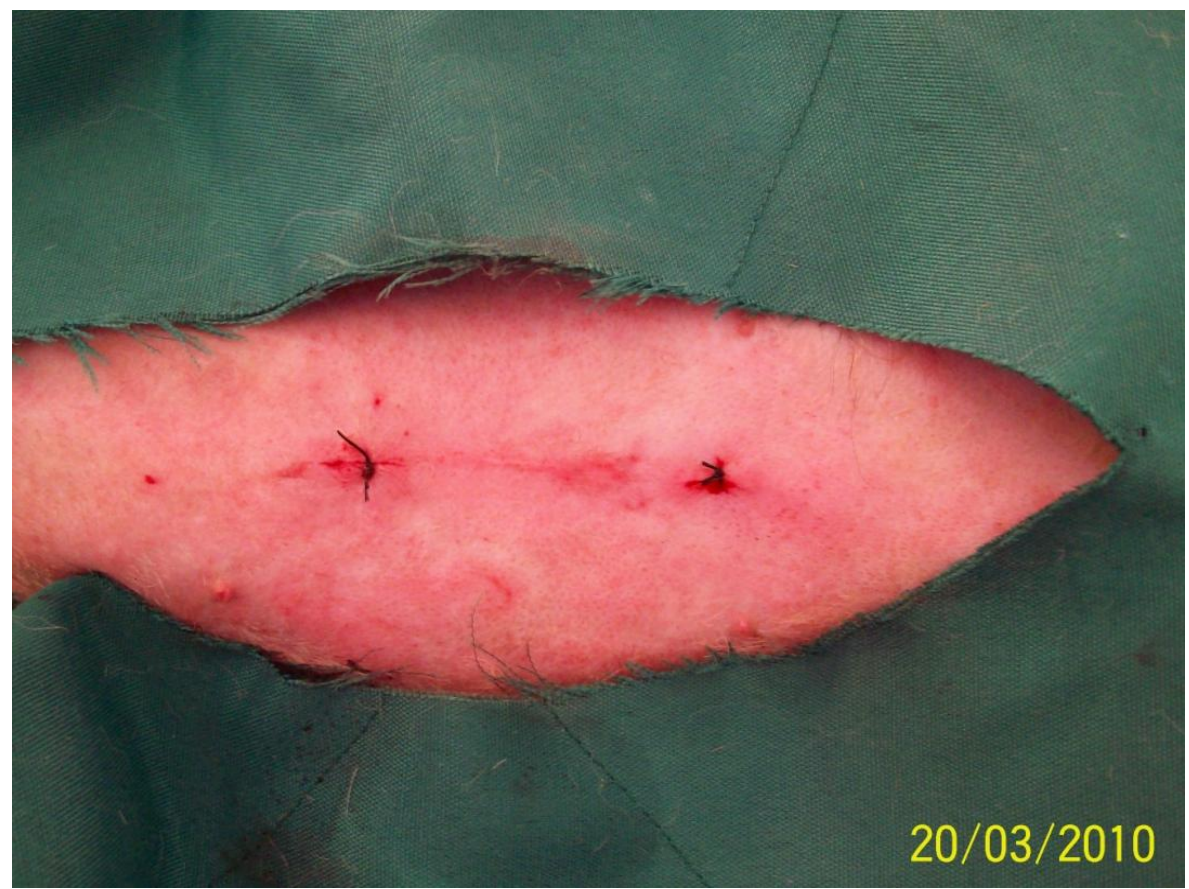

الصورة (7): إغلاق الثقوب بعد الاتتهاء من التظظير

$$
5
$$

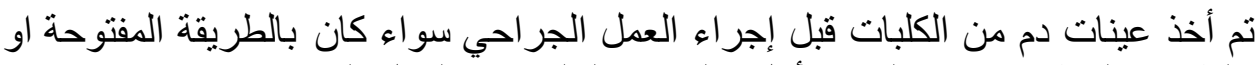

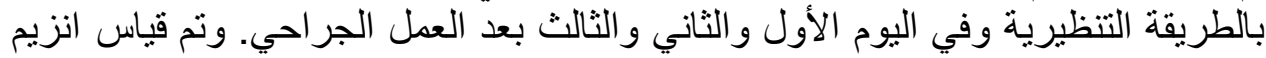

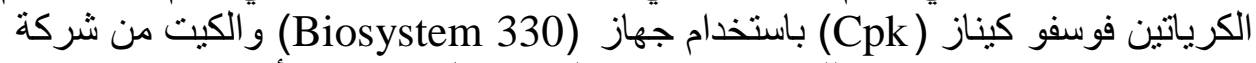
وكذلك تم قياس سكر الدم في كل عينة دم مأخوذة باستخدام نفس (Biomirion، Spain)

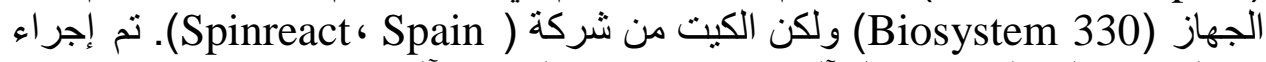
عد الكريات الدم البيض بشكل آلي باستخدام جهاز التعداد الآلي (Sysmex, Japan).

$$
6
$$

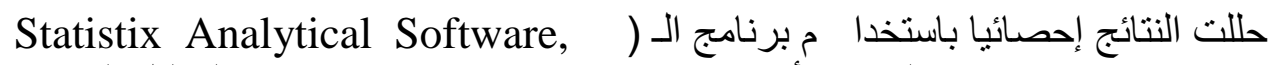

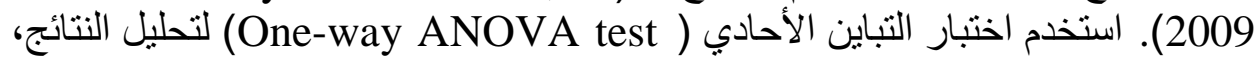

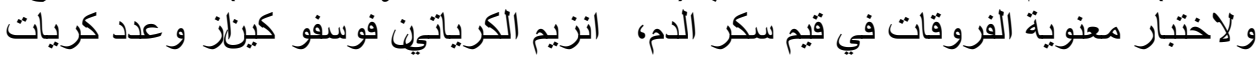

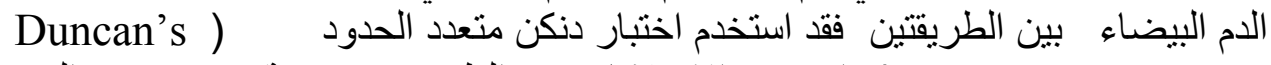
(Multiple Range Test .$(\mathrm{P}<0.05)$

\section{RESULTS}

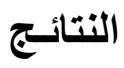




\section{1 - الجراحة التقليدية المفتوحة:}

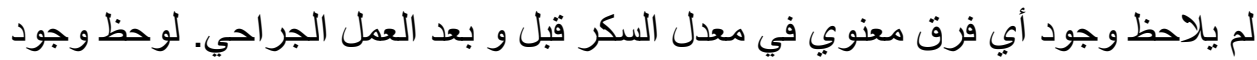

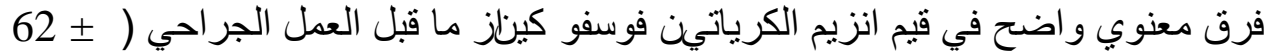

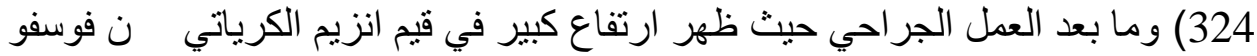

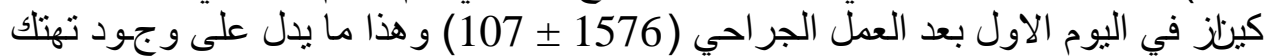

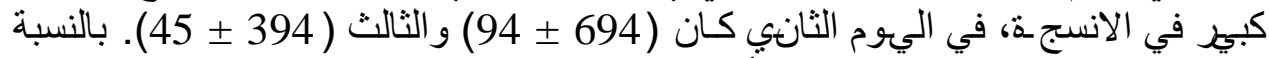

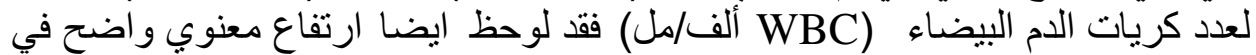

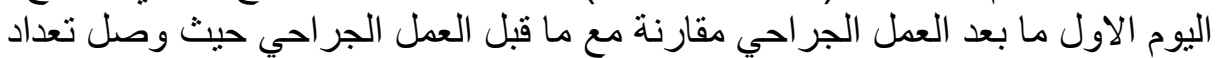

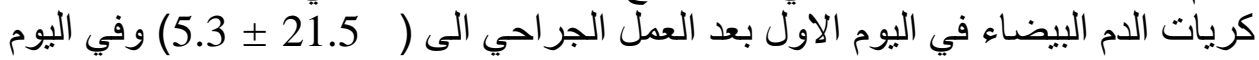

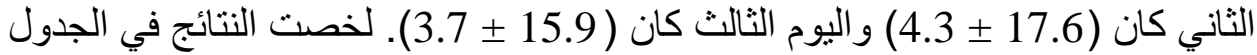

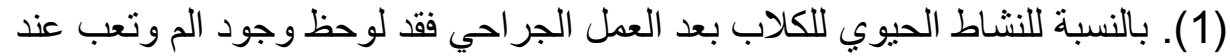
استخدام الجر احة التقليدية الكفتوحة وبقيت الكلاب يولئ العين الجئ حتى استعادت نشاطها الطبيعي.

جدول (1): منوسط السكر وانزيم الكرياتين فوسفو كيناز و عدد الكريات البيض قبل فئل

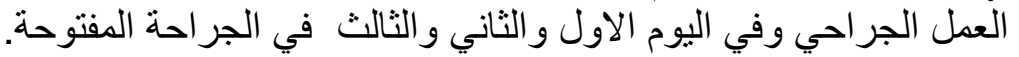

\begin{tabular}{|c|c|c|c|c|c|}
\hline \multirow{2}{*}{ المنوية } & \multicolumn{3}{|c|}{ بعد العمل الجراحي } & \multirow{2}{*}{ قالجر احي } & \\
\hline & اليوم الثالث & اليوم الثاني & اليوم الأول & & \\
\hline NS & $7 \pm 6$ & $7 \pm 64$ & $8 \pm 63$ & $9 \pm 71$ & السكر - السكر \\
\hline$*$ & $45 \pm 394^{\mathrm{d}}$ & $94 \pm 694^{c}$ & $107 \pm 1576^{b}$ & $62 \pm 324^{\mathrm{a}}$ & $\mathrm{Cpk}$ \\
\hline$*$ & $3.7 \pm 15.9^{c}$ & $4.3 \pm 17.6^{c}$ & $5.3 \pm 21.5^{b}$ & $1.9 \pm 12.2^{\mathrm{a}}$ & $\begin{array}{l}\text { ألف:مل } \\
\text { WBC }\end{array}$ \\
\hline
\end{tabular}

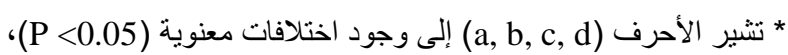
NS = عدم وجود فروق معنوية

2 - 2 - الجراحة التظيرية:

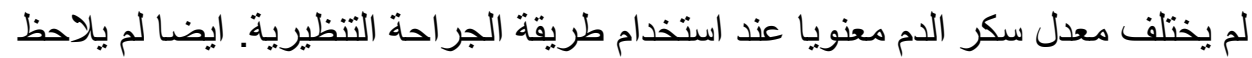

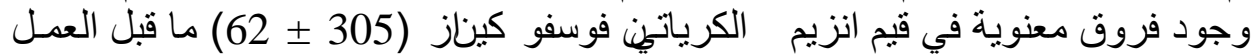

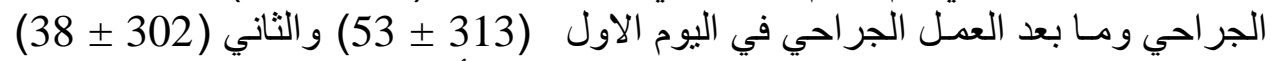

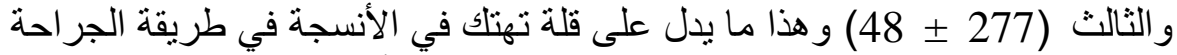

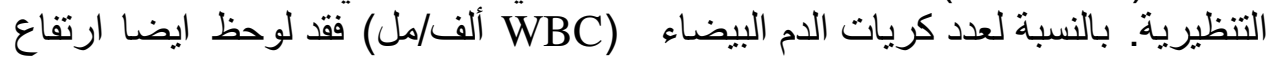

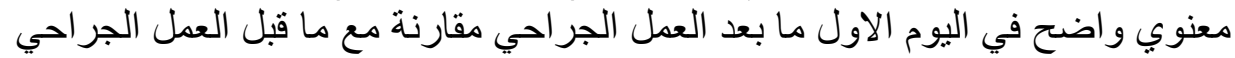

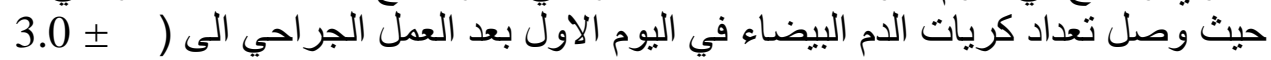

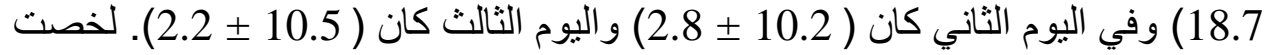

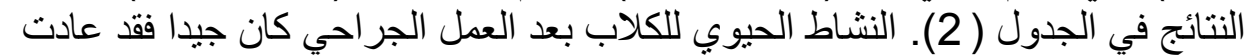
الكلاب التى النشاط الكامل بعد ساعتين من العمل الجراطي لاحئ الجيل 
جدول (2): منوسط السكر وانزيم الكرياتين فوسفو كيناز و عدد الكريات البيض قبل العمل الجر احي وفي اليوم الاول و الثاني والثالث في الجر احة التئن التظيرية.

\begin{tabular}{|c|c|c|c|c|c|}
\hline \multirow{2}{*}{$\begin{array}{l}\text { المعنوية } \\
\text { (Sig) }\end{array}$} & \multicolumn{3}{|c|}{ بعد العمل الجراحي } & \multirow{2}{*}{ قالجر العي } & \\
\hline & اليوم الثالث & اليوم الثاني & اليوم الأول & & \\
\hline NS & $7 \pm 70$ & $11 \pm 73$ & $12 \pm 84$ & $17 \pm 78$ & السكر \\
\hline NS & $48 \pm 277$ & $38 \pm 302$ & $53 \pm 313$ & $62 \pm 305$ & Cpk \\
\hline * & $2.2 \pm 10.5^{\mathrm{c}}$ & $2.8 \pm 10.2^{\mathrm{c}}$ & $3.0 \pm 18.7^{b}$ & $1.8 \pm 13.9^{a}$ & $\begin{array}{l}\text { WBC } \\
\text { ألف/مل }\end{array}$ \\
\hline
\end{tabular}

$$
\begin{aligned}
& \text { * تثبر الأحرف (a, b, c) إلى وجود اختلافات معنوية (P > P) )، } \\
& \text { NS = عدم وجود فروق معنوية }
\end{aligned}
$$

$$
3 \text { - زمن العمل الجراحي: }
$$

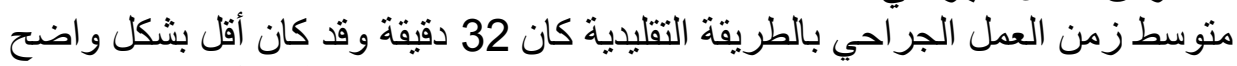

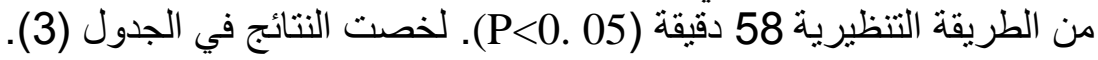

\begin{tabular}{|c|c|c|}
\hline $\begin{array}{l}\text { الجراحة التنظيرية (Laparoscopie) } \\
\text { (Lapale }\end{array}$ & $\begin{array}{c}\text { الجر احة المفتوحة } \\
\text { (Open Surgical) } \\
\end{array}$ & \\
\hline $5 \pm 58$ & $3 \pm 32$ & طول العمل الجر احي \\
\hline
\end{tabular}
جدول ( 3): مدة العمل الجر احي مقرة بالدقيقة بين الجر احة التنظيرية والجر احة

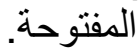

\section{DISCUSSION}

\section{المناقشــة}

الدر اسـات السابقـة قـارنت بيـن التعقيم باستخدام التنظير عند إنات الكـلاب

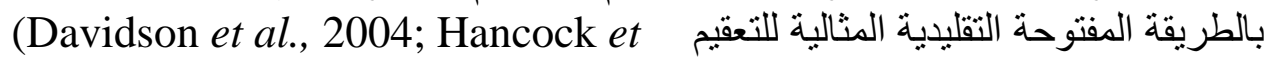
al., 2005; Devitt et al., 2006)

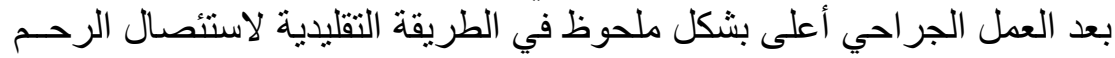

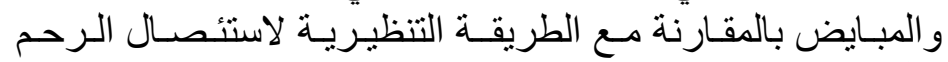

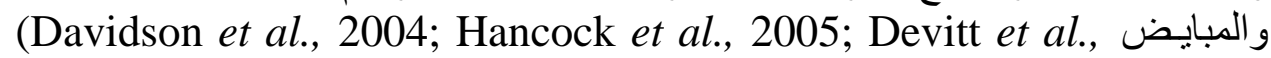

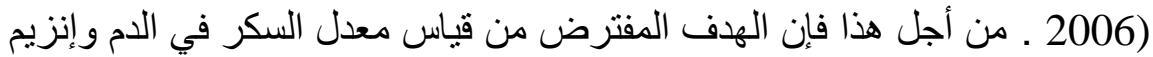

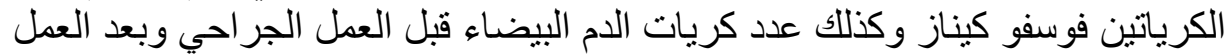
الجر احي لمدة ثلاثة ايام كان لمقارنة الطريقة الجر احة التقليدية مع الجر احة التنظيرية. 
إن بروتوكول التخدير الذي تم استخدامه هو إجر اء شائع الاستخدام في غرفة إنة العمليات الخاصة بالحيو انات الصغيرة والتابعة لجمعية حماية الحيوان التير السبانا في كلية

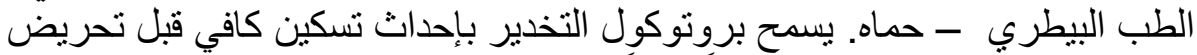

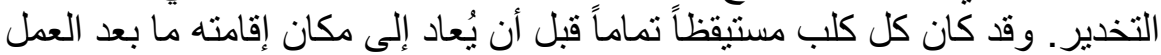

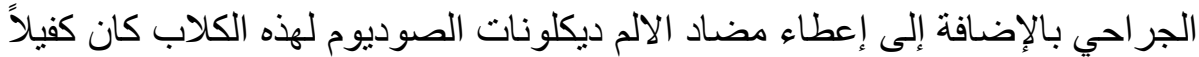

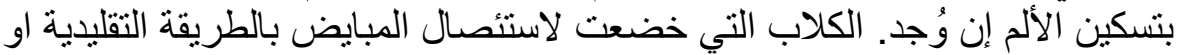
بطريقة الجر احة التنظيرية لم يكن لايها اختلاف معنوي في معدل سكر الإم قبل فيل العمل

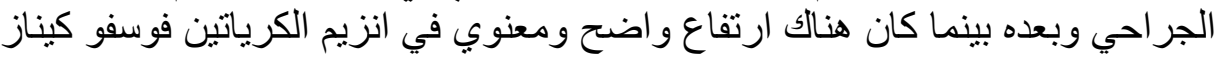

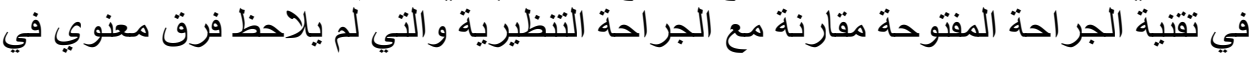

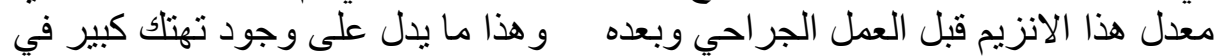

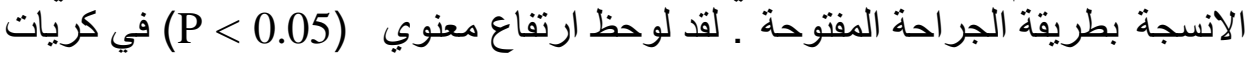

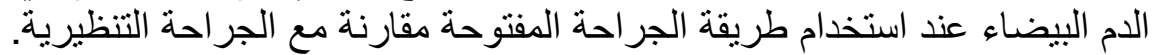

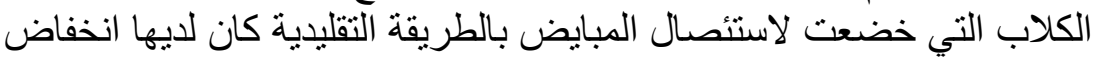

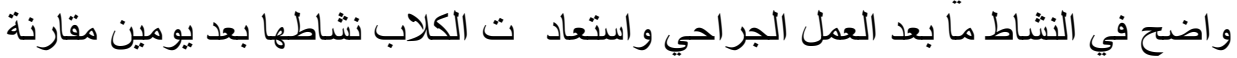

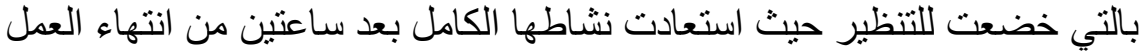

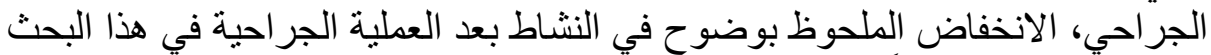

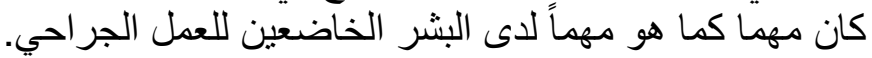
في دراسة قام بها العالم (Inoue et al., 2003) فإن فترة النقاهئ النقاهة كما تم قياسها

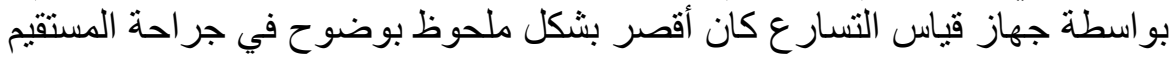

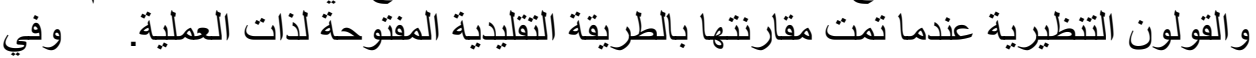

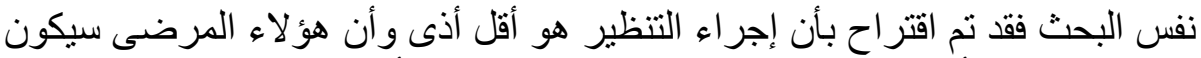

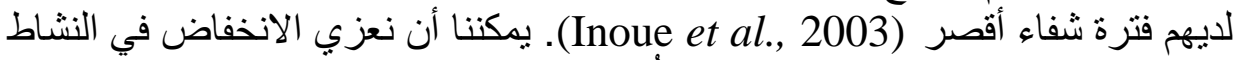

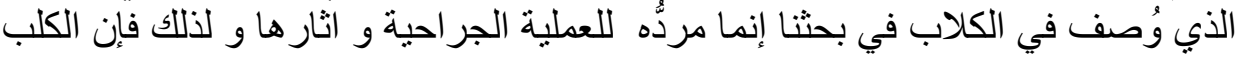

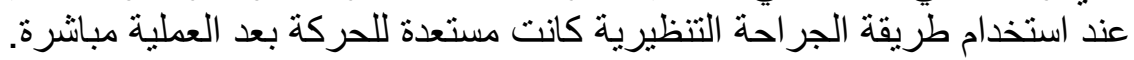

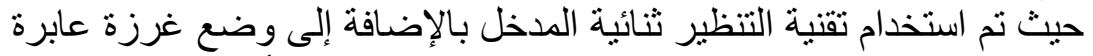

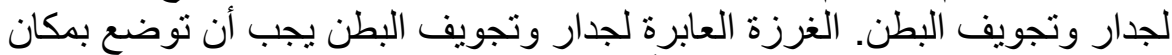

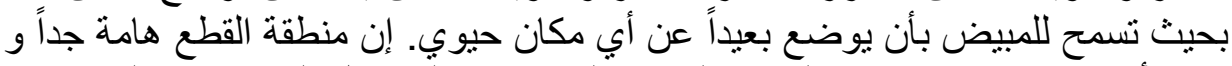

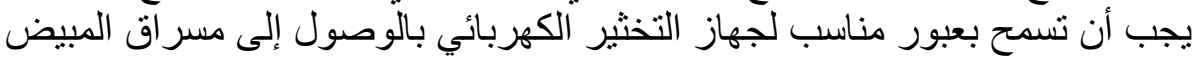

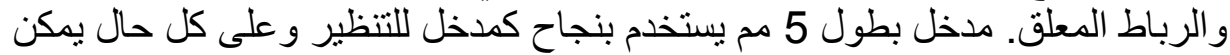

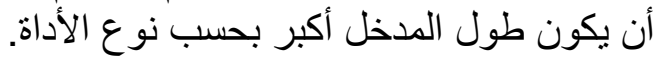

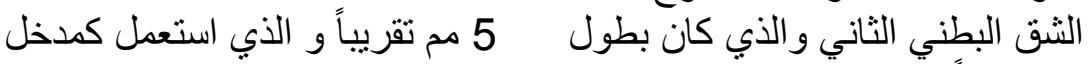

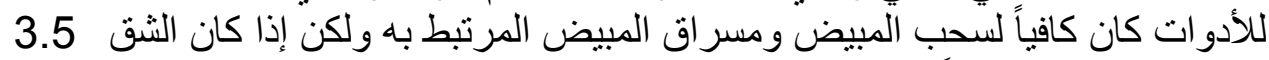

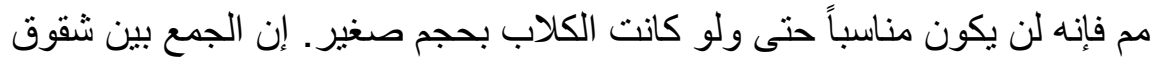

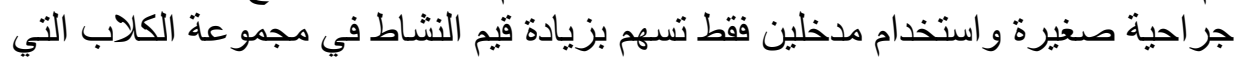

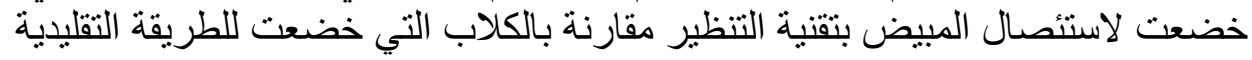

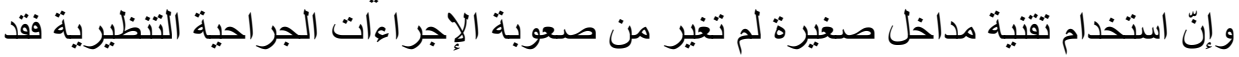


تم استعمال مداخل صغيرة بقطر 3.5 مم عند القطط و الطيور ( Van (Nimwegen et) .al., 2007; Hernandez-Divers et al., 2007) إن جهاز المخثر الكهربائي الذي يعتمد على جهاز تخثير الأوعية الدموية ثنائي

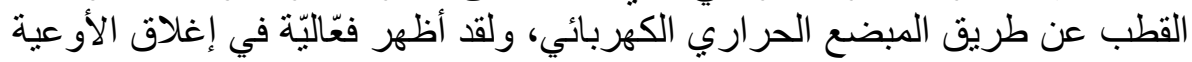

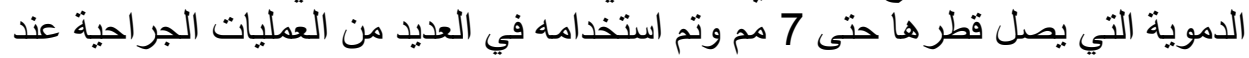
البشر (Soon et al., 2006;Hubner et al., 2007;Kennedy et al., 1998) ولكنه لم يُقيّم في حالات التعقيم الإكلينيكي عند الكلاب. و وعلى كل حال فإن جهاز تخثير

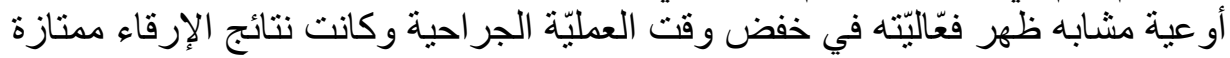

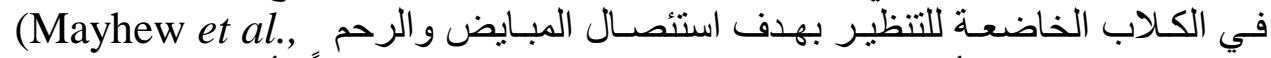
(2007. ولقد وجدنا بأنّ جهاز المخثر الكهربائي سهل الاستخدام جداً وأظهر نتائج مذهلة

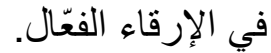

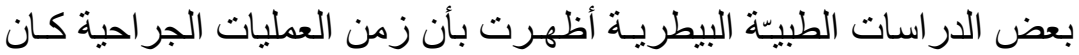

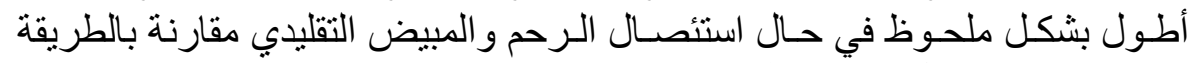

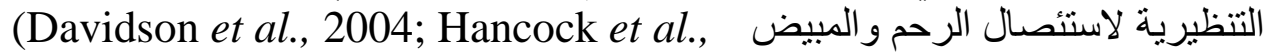

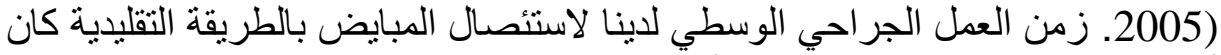
أقصر من الطريقة التنظيرية و على أبي حال فإنّ زمن العملية الجر احيّة الوسطي بالطي الطريقة

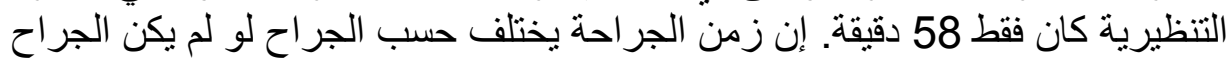

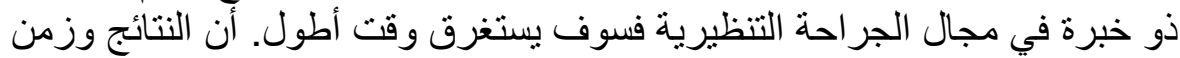

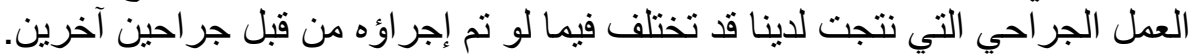

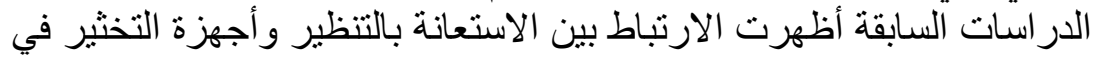

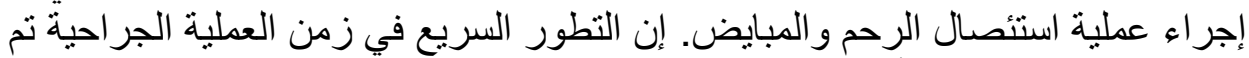

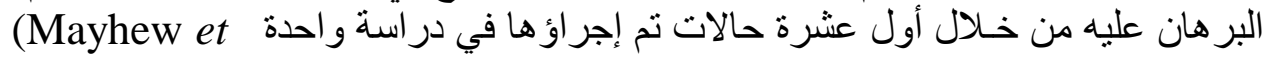

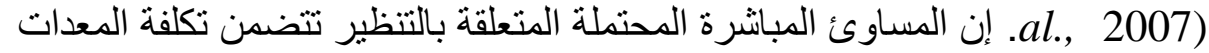

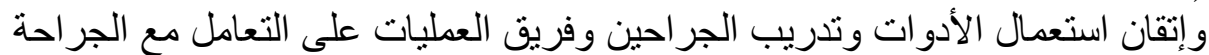

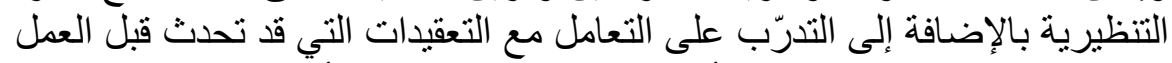

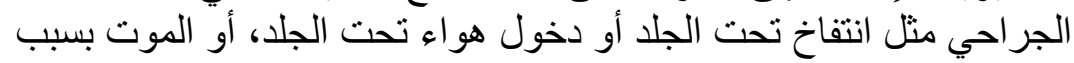
التخدير) (Monnet et al., 2003; Culp et al., 2009)

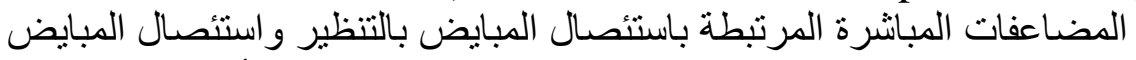

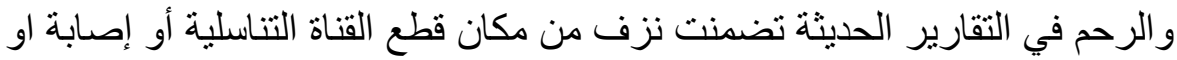
تقرحات عضو ما بالإضافة لمشاكل الجروح مثل التورم و الاحمر ار و الحكة و الانفلاق (Van Goethem et al., 2003; Van Nimwegen et al., 2005; Hancock et (al., 2005; Mayhew et al., 2007)

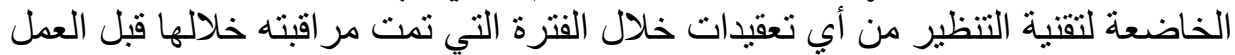

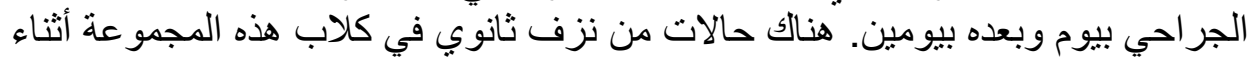
العمل الجر احي ولم يستدعي ذلك أي تدخل للإرقاء أو إيقاف النزيف نديف 


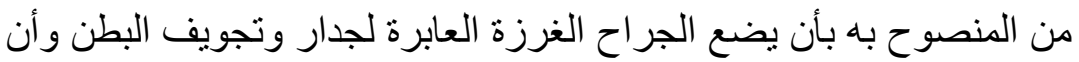

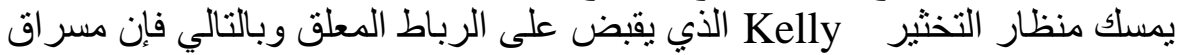

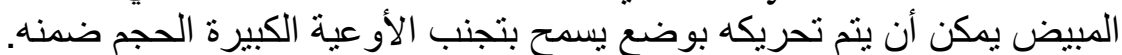

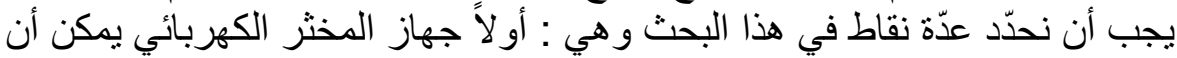

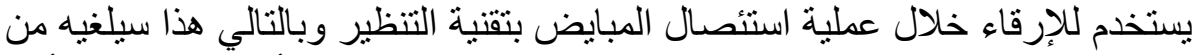
كونه متغير للمقارنة. ضخ غاز CO لدور ضخ

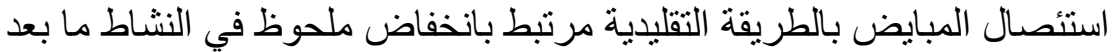

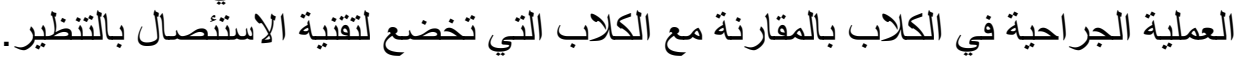

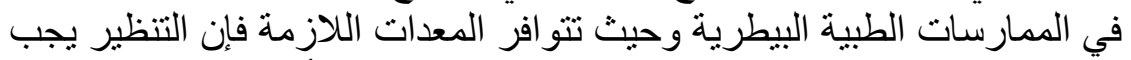

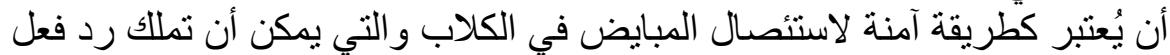
شفائي بعد العملية الجر احية أفضل بكثير من الطريقة التقليدية المفتوحة.

\section{شكر وتقليـر}

يشكر الباحثون شركة كارل سنورز الالمانية (Karl Storz Endoscopy, Goleta, CA) و السيد رياض دعدوش لمساعدته في تقديم الأجهزة والأدول الأدات الجر احة التنظيرية.

\section{REFERENCES المراجع العلمية}

Culp, W.T.N.; Mayhew, P.D. and Brown, D.C. (2009): The Effect of Laparoscopic Versus Open Ovariectomy on Postsurgical Activity in Small Dogs. Veterinary Surgery, 38: 811-817.

Davidson, EB.; Moll, HD. and Payton, ME. (2004): Comparison of laparoscopic ovariohysterectomy and ovariohysterectomy in dogs. Vet. Surg. 33: 62-69.

Devitt, CM.; Cox, RE. and Hailey, JJ. (2005): Duration, complications, stress, and pain of open ovariohysterectomy versus a simple method of laparoscopic-assisted ovariohysterectomy in dogs. J. Am. Vet. Med. Assoc. 227: 921-927.

Inoue, Y.; Kimura, T. and Noro, H. (2003): Is laparoscopic colorectal surgery less invasive than classical open surgery? Quantitation of physical activity using an accelerometer to assess postoperative convalescence. Surg. Endosc. 17: 1269-1273. 
Hancock, RB.; Lanz, OI. and Waldron, DR. (2005): Comparison of postoperative pain after ovariohysterectomy by harmonic scalpel-assisted laparoscopy compared with median celiotomy and ligation in dogs. Vet. Surg. 34: 273-282.

Hernandez-Divers, SJ.; Stahl, SJ. and Wilson, GH. (2007): Endoscopic orchidectomy and salpingohysterectomy of pigeons (Columba livia): an avian model for minimally invasive endosurgery. J. Avian Med. Surg. 21: 22-37.

Hubner, M.; Hahnloser, D. and Hetzer, F. (2007): A prospective randomized comparison of two instruments for dissection and vessel sealing in laparoscopic colorectal surgery. Surg. Endosc. 21: 592-594.

Kennedy, JS.; Stranahan, PL. and Taylor, KD. (1998): Highburststrength, feedback-controlled bipolar vessel sealing. Surg. Endosc. 12: 876-878.

Mayhew, PD. and Brown, DC. (2007): Comparison of three techniques for ovarian pedicle hemostasis during laparoscopic-assisted ovariohysterectomy. Vet. Surg. 36: 541-547.

Monnet, E. and Twedt, DC. (2003): Laparoscopy. Vet. Clin. Small Anim. 33: 1147-1163.

Okkens, AC.; Kooistra, HS. and Nickel, RF. (1997): Comparison of longterm effects of ovariectomy versus ovariohysterectomy in bitches 51(Suppl): 227-231.

Soon, PSH.; Yeh, MW. and Sywak, MS. (2006): Use of the LigaSure vessel sealing system in laparoscopic adrenalectomy. ANZ. J. Surg. 76: 850-852.

Van Goethem, B.; Schaefers-Okkens, A. and Kirpensteijn, J. (2006): Making a rational choice between ovariectomy and ovariohystectomy in the dog: a discussion of the benefits of either technique. Vet. Surg. 35: 136-143.

Van Goethem, BE.; Rosenveldt, KW. and Kirpensteijn, J. (2003): Monopolar versus bipolar electrocoagulation in canine laparoscopic ovariectomy: a nonrandomized, prospective, clinical trial. Vet. Surg. 32: 464-470.

Van Nimwegen, SA.; Van Swol, CFP. and Kirpensteijn, J. (2005):

Neodymium: Yttrium aluminum garnet surgical laser versus 
bipolar electrocoagulation for laparoscopic ovariectomy in dogs. Vet. Surg. 34: 353-357.

Van Nimwegen, SA. and Kirpensteijn, J. (2007): Laparoscopic ovariectomy in cats: comparison of laser and bipolar electrocoagulation. J. Feline Med. Surg. 9: 397-403. 